\title{
Temperature Significantly Affects the Plaquing and Adsorption Efficiencies of Listeria Phages
}

\author{
Jeffrey I. Tokman, David J. Kent, Martin Wiedmann and Thomas Denes* \\ Department of Food Science, Cornell University, Ithaca, NY, USA
}

Listeria-infecting phages are currently being used to control and detect the important foodborne pathogen Listeria monocytogenes; however, the influence of environmental conditions on the interactions between $L$. monocytogenes and its phages has not been explored in depth. Here, we examined the infective potential of four Listeria phages (two each from the P70-like and P100-like phages of Listeria) against five strains of $L$. monocytogenes (representing serotypes 1/2a, 1/2b, 4a, and $4 b$ ) grown under a range of temperatures $\left(7-37^{\circ} \mathrm{C}\right)$. We show that the plaquing efficiencies for all four phages were significantly affected by temperature. Interestingly, no plaques

OPEN ACCESS

Edited by:

Andrea Gomez-Zavaglia,

Center for Research

and Development in Food

Cryotechnology - CONICET,

Argentina

Reviewed by:

Gonçalo Nieto Almeida Universidade Católica Portuguesa,

Portugal

Alexandre Leclercq,

Institut Pasteur, France

${ }^{*}$ Correspondence:

Thomas Denes

tgd32@cornell.edu

Specialty section:

This article was submitted to

Food Microbiology,

a section of the journal

Frontiers in Microbiology

Received: 29 January 2016

Accepted: 18 April 2016

Published: 03 May 2016

Citation:

Tokman Jl, Kent DJ, Wiedmann M and Denes $T$ (2016) Temperature Significantly Affects the Plaquing and Adsorption Efficiencies of Listeria Phages. Front. Microbiol. 7:631. doi: 10.3389/fmicb.2016.00631 were observed for any of the four phages at $37^{\circ} \mathrm{C}$. Adsorption assays performed with the P100-like phages, LP-048 and LP-125, showed that LP-048 had a severely reduced adsorption efficiency against susceptible strains at $37^{\circ} \mathrm{C}$ as compared to $30^{\circ} \mathrm{C}$, suggesting that there is considerably less accessible rhamnose (LP-048's putative phage receptor) on the host at $37^{\circ} \mathrm{C}$ than at $30^{\circ} \mathrm{C}$. LP-125 adsorbed to host cells at $37^{\circ} \mathrm{C}$, indicating that the inability for $L P-125$ to plaque at $37^{\circ} \mathrm{C}$ is not due to adsorption inhibition. LP-048 showed significantly higher adsorption efficiency against a mutant strain lacking $\mathrm{N}$-acetylglucosamine in its wall teichoic acids (WTA) than the parental strain at both 30 and $37^{\circ} \mathrm{C}$, suggesting that $\mathrm{N}$-acetylglucosamine competes with rhamnose for glycosylation sites on the WTA. The data presented here clearly shows that $L$. monocytogenes can gain physiological refuge from phage infection, which should be carefully considered for both the design and implementation of phage-based control and detection applications.

Keywords: bacteriophages, Listeria monocytogenes, food safety, teichoic acids, phage-resistance, physiological refuge

\section{INTRODUCTION}

Listeria monocytogenes is the foodborne pathogen that causes listeriosis, which has a mortality rate of 20-30\% (Vazquez-Boland et al., 2001) and causes an estimated 255 deaths per year in the US (Scallan et al., 2011). It has been estimated that the total annual cost of L. monocytogenes in the US is $\sim \$ 2.5$ billion (Batz et al., 2012). A recent meta-analysis on the global burden of listeriosis found that there is an estimated 23,150 human incidents of listeriosis every year (de Noordhout et al., 2014). Outbreaks caused by L. monocytogenes are a major concern for the food industry; between 1998 and 2008 there have been 24 confirmed outbreaks of listeriosis in the US, which resulted in a total of 359 illnesses, 215 hospitalizations and 38 deaths (Cartwright et al., 2013). 
Since 2006, lytic bacteriophages (or "phages") have been used according to national or regional regulations as biocontrol agents that specifically target L. monocytogenes. Studies that have evaluated the efficacy of phage-based biocontrol of L. monocytogenes have shown promise for this listeriocidal application (Strydom and Witthuhn, 2015); however, several obstacles still need to be overcome to improve the long-term efficacy of phage-based biocontrol of L. monocytogenes. For example, the influence of environmental conditions on the susceptibility of L. monocytogenes to phage infection has not been explored in depth (Denes and Wiedmann, 2014). A study by Kim and Kathariou (2009) showed that typically phagesensitive epidemic clone II strains of $L$. monocytogenes were broadly resistant to phage infection at temperatures below $30^{\circ} \mathrm{C}$. A follow-up study found that the temperature-dependent phage resistance was due to a restriction modification system that was expressed at lower temperatures (Kim et al., 2012); however, it is not known if other strains have similar temperature dependent mechanisms of phage-resistance.

Here, we examined how temperature affects the infection potential of Listeria-infecting phages against a representative panel of L. monocytogenes strains. Our goal was to advance our understanding of how environmental conditions affect phagesusceptibility of Listeria by focusing on the key condition, temperature. Further, this study was designed to provide knowledge that can improve the implementation and design of phage-based biocontrol and detection applications.

\section{MATERIALS AND METHODS}

\section{Phages and Bacterial Strains}

Two phages each from the obligate lytic phage groups P100like phages (phages LP-048 and LP-125) and P70-like phages (LP-026 and LP-037) were selected for use in this study (Denes et al., 2014) [Table 1]. We also included Listeria phage A511 (Table 1) so that we could make comparisons to other studies. The L. monocytogenes strains used in this study were selected to represent a diversity of lineages (Lineage I, II, and IV) and serotypes (1/2a, 1/2b, 4a, and 4b; Table 1). The P100-like phages used in this study were propagated on Mack and the P70-like phages used in this study were propagated on FSL J1-208 (also the isolation host) as previously described (Vongkamjan et al., 2012). The plasmid cured strain of FSL J1-208, designated FSL B2-294, was also included in this study to test whether the plasmid is linked to phage susceptibility (Table 1). The strains 541-NM, 542-NM, and $\Delta$ dltA (Table 1) were used in this study because the changes to their surface structures are known to affect (Denes et al., 2015), or may affect, phage adsorption. Strains 541-NM-C and 542-NM-C were included (Table 1) to show that complementation of the mutations in 541-NM and 542-NM with the wild-type alleles of LMRG_00541 and LMRG_00542, respectively, rescues the wild-type phenotype.

\section{Efficiency of Plaquing Assays}

Bacterial lawns were prepared using a double agar overlay method (Kropinski et al., 2009) using modified LB MOPS media (at a $\mathrm{pH}$ of 7.4) as previously described (Vongkamjan et al., 2012). A $30 \mu l$ aliquot of $L$. monocytogenes culture grown overnight $(16 \pm 2 \mathrm{~h})$ in BHI broth (shaking at $210 \mathrm{RPM}$ ) was used to seed each lawn. Immediately after bacterial lawns were poured the plates were acclimated for 60 to $90 \mathrm{~min}$ at the experimental temperatures before phage dilutions were spotted on the acclimated lawn. Phage dilutions that were spotted on plates ranging in concentration from $2 \times 10^{2}$ to $3 \times 10^{9} \mathrm{PFU} / \mathrm{mL}$. Immediately after spotting, the plates were incubated at the experimental temperatures. Temperatures were selected to represent conditions phage-based products would be expected to function under; for example, $6.5^{\circ} \mathrm{C}$ is a temperature that some refrigerated foods may be held at, and is similar to the $7^{\circ} \mathrm{C}$ temperature used by Kang et al. (2014) in Listeria growth studies on cold-smoked salmon. Plates incubated at $21 \pm 1$, $25 \pm 1,30 \pm 2$, and $37 \pm 1^{\circ} \mathrm{C}$ were incubated for $16 \pm 2 \mathrm{~h}$ before enumeration. Plates incubated at $12 \pm 1$ and $16.5 \pm 1.5^{\circ} \mathrm{C}$ were incubated for $40 \pm 2 \mathrm{~h}$ before enumeration. Plates incubated at $6.5 \pm 1.5^{\circ} \mathrm{C}$ were incubated for 7 days before enumeration. Longer incubation times at lower temperatures were needed to allow for visible plaques to form. Only visible plaques were counted; faint turbidity without plaque formation was scored as zero.

\section{Adsorption Assays}

LP-048 and LP-125 adsorption assays were performed by following a modified version of a previously described procedure (Denes et al., 2015). Modifications were made to adapt the procedure to compare adsorption at different experimental temperatures $\left(30^{\circ} \mathrm{C}\right.$ vs. $\left.37^{\circ} \mathrm{C}\right)$. Briefly, overnight cultures (incubated for $16 \pm 2 \mathrm{~h}$ ) were grown in BHI broth at the experimental temperatures with shaking at 210 RPM and then diluted 1/100 into fresh BHI broth. The diluted cultures were then incubated at their respective experimental temperature $\left(30^{\circ} \mathrm{C}\right.$ or $37^{\circ} \mathrm{C}$ ) with shaking at $210 \mathrm{RPM}$ until they reached an $\mathrm{OD}_{600}$ of 0.4 . Then, $200 \mu \mathrm{l}$ volumes of cultures (at an $\mathrm{OD}_{600}$ value of 0.4 ) were transferred into microcentrifuge tubes containing $762 \mu \mathrm{l}$ BHI broth, $20 \mu \mathrm{l}$ phage lysate at $1 \times 10^{9} \mathrm{PFU} / \mathrm{mL}, 9 \mu \mathrm{l}$ of $1 \mathrm{M}$ $\mathrm{CaCl}_{2}$, and $9 \mu \mathrm{l}$ of $1 \mathrm{M} \mathrm{MgCl}_{2}$ (salts were added immediately prior to the addition of bacteria). The bacteria and phage mixture was then incubated for $15 \mathrm{~min}$ at either $30^{\circ} \mathrm{C}$ or $37^{\circ} \mathrm{C}$ with aeration.

\section{Statistical Analyses}

All statistical analyses were carried out in R (V.3.0.3; R Core Team, 2009). Data handling was performed using reshape (Wickham, 2007). Linear models were constructed using the $\mathrm{lm}$ command from the base package. Pairwise comparisons at all factor levels were made using lsmeans with Tukey multiple testing correction (Lenth and Hervé, 2014). The cutoff for significance was set at $\alpha=0.05$.

Models for efficiency of plaquing (EOP) experiments and models for adsorption assay experiments had the factors strain, temperature, and biological replicate, as well as the 2-way interaction between strain and temperature. Relative EOP values were the model responses for plaquing experiments, whereas the $\log _{10}$ reductions of PFU in the supernatant were the model responses for adsorption assays. 
TABLE 1 | Phages and bacterial strains used in study.

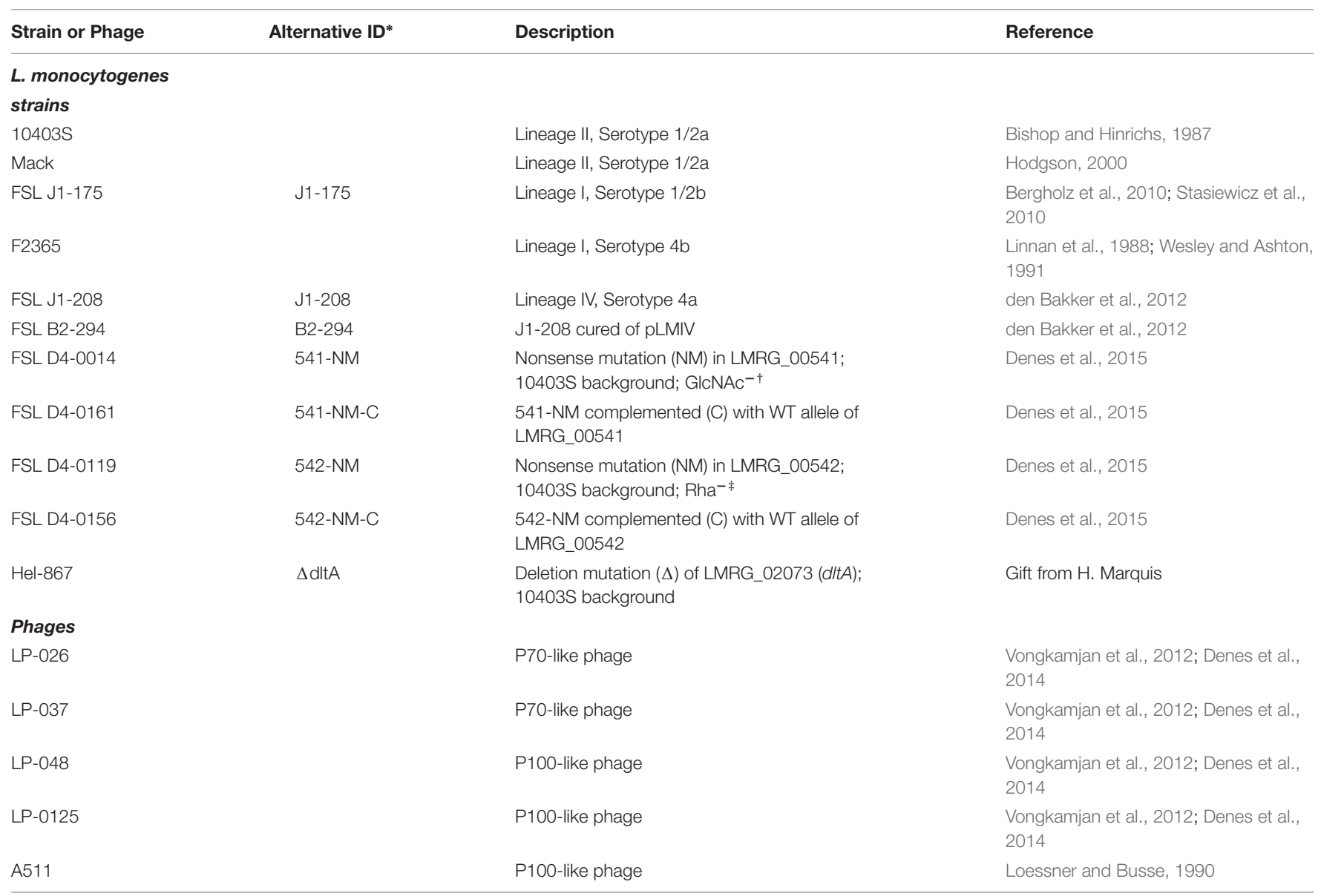

*Strain ID used in the manuscript text and figures. ${ }^{\dagger}$ The wall teichoic acids of the indicated strain lack N-acetylglucosamine (GIcNAc). *The wall teichoic acids of the indicated strain lack rhamnose (Rha).

\section{BLAST Searches}

BLAST searches (Altschul et al., 1990) were conducted against the L. monocytogenes 10403S (GenBank accession no. NC_017544.1), F2365 (GenBank accession no. NC_002973.6), and J1-208 (GenBank accession no. NZ_CM001469.1) genomes with the protein query sequences for the LmoH7 restriction-modification system (GenBank accession nos. EAL08857.1 and EAL08858.1). We defined our cutoff for homology as $25 \%$ shared sequence identity with $\geq 50 \%$ query coverage.

\section{Data and Script Availability}

The raw data and $\mathrm{R}$ scripts used in this study can be found in the Supplementary File 1.

\section{RESULTS}

\section{Spot Assays Reveal That Temperature Affects Phage Infection of \\ L. monocytogenes}

Initial spot testing of phages A511, LP-125, and LP-048 revealed no visible plaques on Mack or F2365 at $37^{\circ} \mathrm{C}$, although faint clearings were observed where the most concentrated phage solutions were spotted (Figure 1). All three phages formed visible plaques on Mack at $30^{\circ} \mathrm{C}$ (Figure 1); however, only LP-125 and A511 formed visible plaques on $\mathrm{F} 2365$ at $30^{\circ} \mathrm{C}$. The EOP of LP-125 and A511 at $30^{\circ} \mathrm{C}$ was lower on F2365 than on Mack (Figure 1).

\section{Efficiency of Plaquing of P100-like Phages Is Affected by Growth Temperature}

Quantitative plaquing assay data collected at six different temperatures with five different host strains and two different P100-like phages (LP-048 and LP-125) were used to statistically evaluate the effects of host and temperature on plaquing. For LP-048, plaquing efficiency was significantly affected by both strain $(p<0.001)$ and temperature $(p<0.001)$; there was also a significant interaction between strain and temperature $(p<0.001$; see Supplementary File 1). LP-048 formed visible plaques on 10403S, Mack, and J1-175 at all temperatures except $37^{\circ} \mathrm{C}$, but did not form visible plaques on F2365 and J1208 at any temperature (Figure 2A). Effects of temperature on plaque formation were similar for $10403 \mathrm{~S}$ and Mack; 


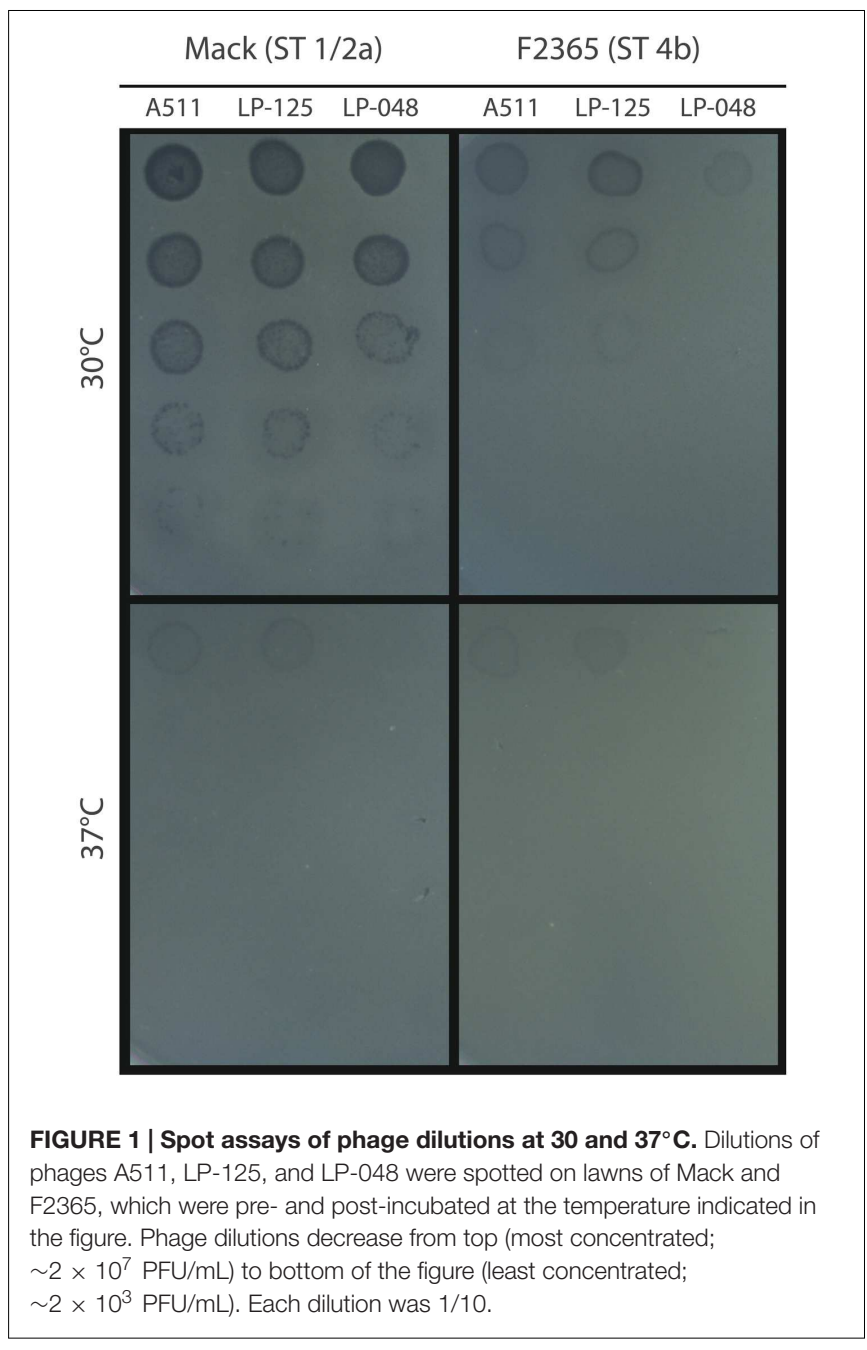

LP-048 showed a trend of having a higher EOP at lower temperatures (Figure 2A) with numerically largest EOPs at $12^{\circ} \mathrm{C}$ and significantly lower EOPs at higher temperatures for both serotype 1/2 host strains (Figure 2A). Interestingly, temperature dependent patterns of plaque formation on the host strain J1175 were different; on this host strain, the EOP values of LP048 were significantly lower at 12 and $21^{\circ} \mathrm{C}$ as compared to the EOP values at $6.5,25$, and $30^{\circ} \mathrm{C}$ (Figure 2A). This may suggest there is a mechanism of phage-resistance in J1-175 (e.g., a restriction modification system) that is only expressed at temperatures between 12 and $21^{\circ} \mathrm{C}$. This would be consistent with the previously described temperature-dependent restriction modification system found in epidemic clone II strains of L. monocytogenes, which is only active at temperatures below $30^{\circ} \mathrm{C}$ (Kim et al., 2012).

For LP-125, plaquing efficiency was significantly affected by both strain $(p<0.001)$ and temperature $(p<0.001)$; there was also a significant interaction between strain and temperature $(p<0.001$; see Supplementary File 1$)$. LP-125 formed visible plaques on 10403S, Mack, and F2365 at all temperatures except $37^{\circ} \mathrm{C}$, but did not form visible plaques on J1-175 and J1-208 at any temperature (Figure 2B). Effects of temperature on plaque formation were similar for 10403S and Mack; LP-125 showed a trend of having a higher EOP at lower temperatures (Figure 2B) with numerically largest EOPs at $12^{\circ} \mathrm{C}$ and significantly lower EOPs at $30^{\circ} \mathrm{C}$ for both host strains (Figure 2B). Interestingly, temperature dependent differences in plaque formation were more pronounced on F2365; on this host strain the EOP was significantly higher at $12^{\circ} \mathrm{C}$ (as compared to all other temperatures), and the EOP was significantly lower at $30^{\circ} \mathrm{C}$ than at temperatures between 6.5 and $25^{\circ} \mathrm{C}$ (Figure 2B).

\section{Efficiency of Plaquing of P70-like Phages Is Affected by Growth Temperature}

Quantitative plaquing assay data collected at six different temperatures with three different host strains and two different P70-like phages (LP-026 and LP-037) were used to statistically evaluate the effects of host and temperature on plaquing. Host strains Mack, J1-175, and F2365 were not used because P70like phages have a narrow host range as compared to P100like phages (Vongkamjan et al., 2012). For LP-026, plaquing efficiency was significantly affected by both strain $(p<0.001)$ and temperature $(p<0.001$; see Supplementary File 1$)$. LP026 formed visible plaques on J1-208, B2-294, and $10403 \mathrm{~S}$ at all tested temperatures except at $37^{\circ} \mathrm{C}$ (Figure 3A). Effects of temperature on plaquing were similar for J1-208 and B2-294; LP026 showed a general trend of infecting at a higher EOP at lower temperatures, with the numerically highest $\mathrm{EOP}$ at $16^{\circ} \mathrm{C}$ for both strains, and significantly lower EOPs at $30^{\circ} \mathrm{C}$ than at $16^{\circ} \mathrm{C}$ for B2-294 (Figure 3A). On strain 10403S, the EOP of LP-026 was numerically highest at $16^{\circ} \mathrm{C}$; however, no significant differences were observed between the tested temperatures (Figure 3A).

For LP-037, plaquing efficiency was significantly affected by both strain $(p<0.001)$ and temperature $(p<0.001)$; there was also a significant interaction between strain and temperature ( $p<0.001$; see Supplementary File 1). LP-037 formed visible plaques on J1-208, B2-294, and 10403S at all tested temperatures except for $37^{\circ} \mathrm{C}$ (Figure 3B). Effects of temperature on plaquing were similar for J1-208 and B2-294; LP-037 showed a general trend of infecting at a higher EOP at lower temperatures, with the numerically highest $\mathrm{EOP}$ at $16^{\circ} \mathrm{C}$, and significantly lower EOPs at $30^{\circ} \mathrm{C}$ than at $16^{\circ} \mathrm{C}$ for each strain (Figure 3B). On strain 10403S, the EOP of LP037 was numerically highest at $16^{\circ} \mathrm{C}$; however, no significant differences were observed between the tested temperatures (Figure 3B).

Neither LP-026 nor LP-037 showed any significant differences in EOP between J1-208 and the plasmid cured strain of J1-208, B2-294 (Figure 3). This suggests that the plasmid harbored by J1208, which is unique amongst $L$. monocytogenes in gene content (den Bakker et al., 2012), does not have an effect on the EOP of the P70-like phages.

\section{Adsorption Efficiencies of LP-048 and LP-125 Are Affected by Temperature}

As no visible plaque formation was observed at $37^{\circ} \mathrm{C}$, we set out to determine whether adsorption was inhibited at this 
A

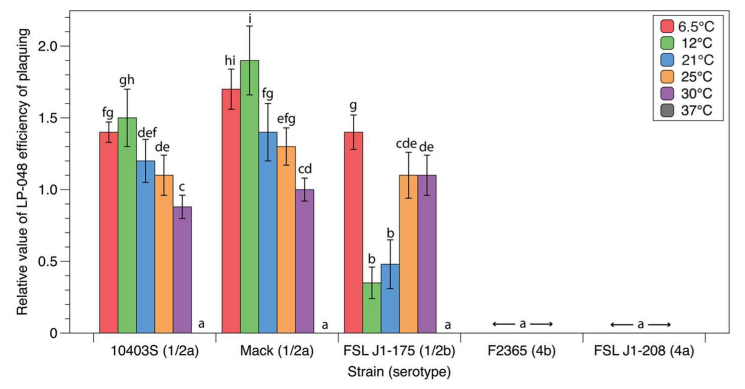

B

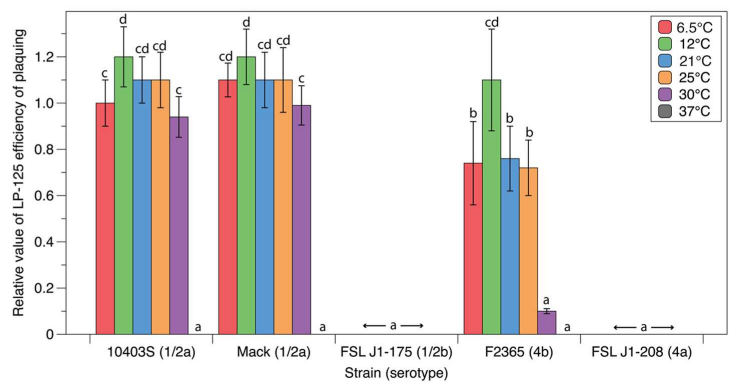

FIGURE 2 | Plaquing efficiencies of P100-like phages at varying growth temperatures. The relative number of visible plaques under different strain and temperature conditions were compared for phages (A) LP-048 and (B) LP-125. All values are relative to the phages isolation conditions (strain Mack at $30^{\circ} \mathrm{C}$ ). Bars that do not share any letters represent values that are significantly different; for example, a bar marked with $\mathrm{d}$, e, and fis significantly different from a bar marked with $\mathrm{g}$ and $\mathrm{h}$. Three biological replicates were conducted. Error bars represent the standard error.

temperature. For these experiments, we used the P100-like phages LP-125 and LP-048 as we have previously identified their putative binding receptors (Denes et al., 2015). The adsorption assays showed, based on the $\log _{10}$ reduction of free phage after coincubation with bacteria, that LP-048 had significantly lower adsorption efficiencies at $37^{\circ} \mathrm{C}$ than at $30^{\circ} \mathrm{C}(0.01$ to 0.03 vs. 0.62 to $1.1 \log$ reduction) on the strains observed to be susceptible to LP-048 (10403s, Mack, and J1-175; Figure 4A). We did not observe LP-048 adsorption at $30^{\circ} \mathrm{C}$ or $37^{\circ} \mathrm{C}$ on the strains where LP-048 plaquing was not seen (J1-208 and F2365; Figure 4A). Interestingly, LP-125 showed nearly the same adsorption efficiencies at both 30 and $37^{\circ} \mathrm{C}$ on the serotype 1/2 strains 10403s, Mack, and J1-175; however, the adsorption efficiency of LP-125 on strain F2365 was significantly lower at $37^{\circ} \mathrm{C}$ than at $30^{\circ} \mathrm{C}(0.18$ vs. $0.83 \log$ reduction; Figure $4 \mathbf{B})$. It should also be noted that LP-125 did adsorb to the strain J1175 (Figure 4B), despite not forming any visible plaques on the strain (Figure 2B). This suggests that a phage resistance mechanism other than adsorption inhibition is preventing LP125 from successfully infecting strain J1-175. Interestingly, this resistance mechanism is not effective against LP-048, which shares a high nucleotide similarity to LP-125 (Denes et al., 2014).

\section{Adsorption Efficiency of LP-048 Is Increased in a Mutant Strain Lacking GIcNAc As a Wall Teichoic Acid Sugar}

LP-048 requires rhamnose biosynthesis genes in its host for adsorption (Denes et al., 2015), these genes have since been confirmed to be essential for rhamnosylation of wall teichoic acids (WTA; Carvalho et al., 2015). We thus formulated two competing hypotheses: (i) that down-regulation of rhamnose expression on the WTA is responsible for the reduced adsorption efficiency of LP-048 observed at $37^{\circ} \mathrm{C}$, or (ii) that up-regulation of GlcNAc expression on the WTA is responsible for the reduced adsorption efficiency of LP-048 observed at $37^{\circ} \mathrm{C}$ [as GlcNAc likely competes for the same sites on the polyol phosphate backbone of the WTA (Fiedler, 1988)]. We show here that LP-048 still adsorbs more efficiently against the mutant strain without GlcNAc in its WTA (541-NM) at $30^{\circ} \mathrm{C}$ than at $37^{\circ} \mathrm{C}$ (no significant difference of the effect of temperature between 10403 S and 541-NM). This supports the first hypothesis that rhamnosylation of WTA is likely being directly down-regulated at $37^{\circ} \mathrm{C}$. We also show that LP-048 does adsorb significantly more efficiently against the 541-NM mutant than against the parental strain at both $30^{\circ} \mathrm{C}(1.55$ vs. $0.55 \mathrm{log}$ reduction) and $37^{\circ} \mathrm{C}$ ( 1.08 vs. $0.19 \log$ reduction; Figure 5A). This suggests that there is competition between rhamnose and $N$-acetylglucosamine glycosylation sites on the WTA (discussed further below); however, our data do not support that up-regulation of GlcNAc expression on the WTA is responsible for the reduced adsorption efficiency of LP-048 at $37^{\circ} \mathrm{C}$. Consistent with our previous results (Denes et al., 2015), LP-048 and LP-125 showed severely reduced adsorption to the rhamnose deficient WTA mutant, 542-NM, and LP-125 showed severely reduced adsorption to 541-NM (Figure 5). Complementation of 541-NM and 542NM with their respective wild-type alleles (i.e., LMRG_00541 and LMRG_00542) restored the strains' observed phenotypes to those of the respective parental strain (10403S; Figure 5). We also tested adsorption of phages LP-048 and LP-125 at 30 and $37^{\circ} \mathrm{C}$ against the strain $\Delta$ dltA, which is incapable of incorporating $\mathrm{D}$-alanine onto its teichoic acids; no significant differences were observed between $\Delta$ dltA and its parental strain (Figure 5).

\section{No Homologous Genes to LmoH7 Were Found in the Host Strains Used in This Study}

Our observation that LP-125 does still adsorb at $37^{\circ} \mathrm{C}$ suggests that there is an adsorption-independent mechanism of phage resistance that is preventing plaque formation at $37^{\circ} \mathrm{C}$. We performed BLAST searches against the genomes of strains 10403S, J1-208, and F2365 (the host strains with available genome sequences) to see if any genes homologous to the temperature dependent LmoH7 restriction-modification system found in epidemic clone II strains of L. monocytogenes (Kim et al., 2012) were present. No homology was found, which suggests that the 


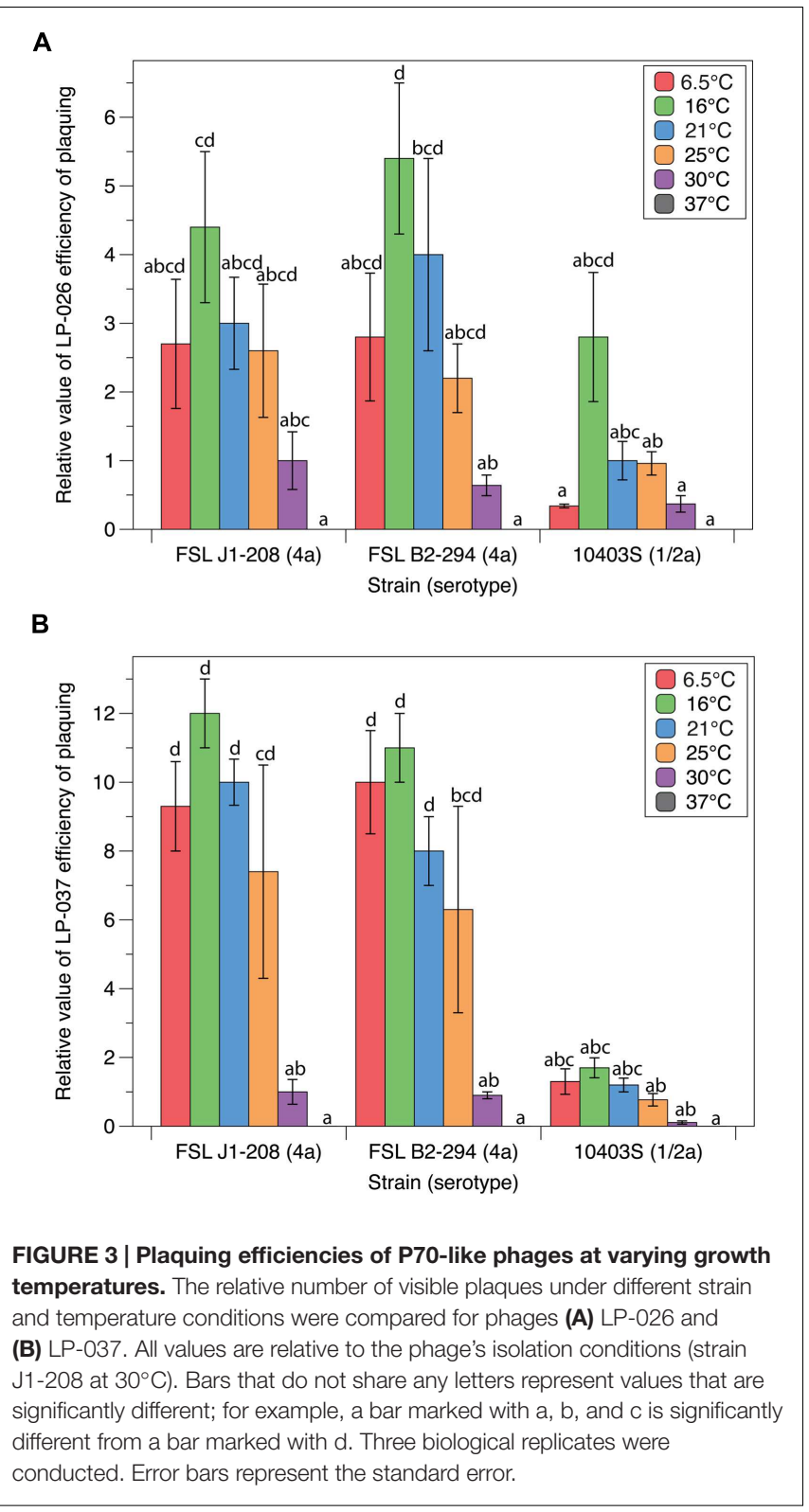

adsorption-independent mechanism of phage resistance we have observed in this study is different from that caused by LmoH7.

\section{DISCUSSION}

In this study, we evaluated the effect of temperature on the infection process of four Listeria phages, representing two distinct phage groups. We showed that (i) EOP is significantly affected by temperature, most notably that no plaques were observed under any phage-host combination at $37^{\circ} \mathrm{C}$, that (ii) adsorption efficiency is affected by temperature in specific cases, but does not explain the general lack of observed plaque formation at $37^{\circ} \mathrm{C}$, and that (iii) there is competition between rhamnose and GlcNAc glycosylation of the WTA at both 30 and $37^{\circ} \mathrm{C}$ (which affects phage adsorption). Our data clearly showed that
A
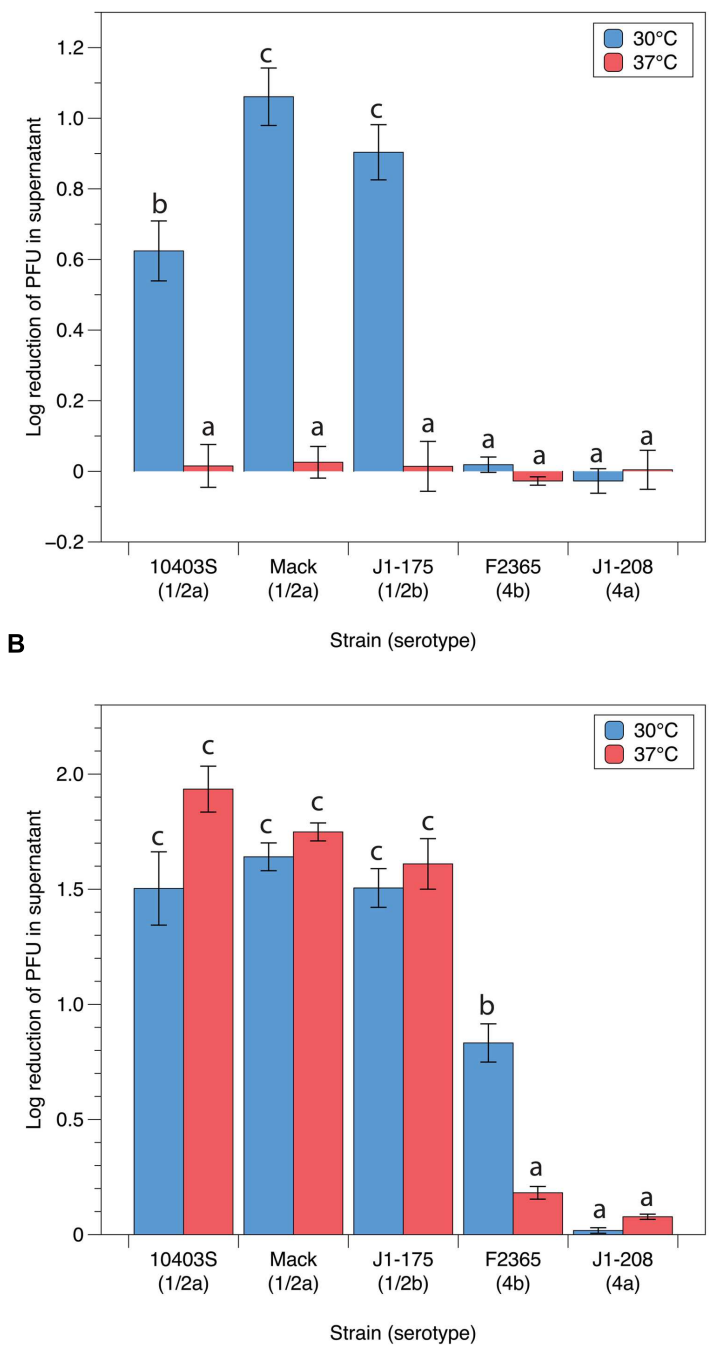

FIGURE 4 | Adsorption efficiencies of P100-like phages (A) LP-048 and (B) $\mathbf{L P}-125$ at $30^{\circ} \mathbf{C}$ and $37^{\circ} \mathbf{C}$. Values shown are the $\log _{10}$ reduction of free phage in the supernatant of each co-incubation between phage and host. The $\log _{10}$ reduction is calculated by subtracting the log-transformed number of phage left in in the supernatant from the log-transformed number of phage in the negative control (BHI). Lower values represent less efficient adsorption. Bars that do not share any letters represent values that are significantly different; for example, a bar marked with $\mathrm{b}$ is significantly different from a bar marked with c. Three biological replicates were conducted. Error bars represent the standard error.

L. monocytogenes can gain physiological refuge from phage infection (i.e., transient resistance), which should be carefully considered when selecting combinations of phages for use in biocontrol or detection applications (Denes and Wiedmann, 2014). These data are practically important as phage-based applications for Listeria may need to function under a range of temperatures; for example, biocontrol tools used in food safety will likely need to work at both refrigeration and ambient temperatures, and detection assays may be expected to function 
A

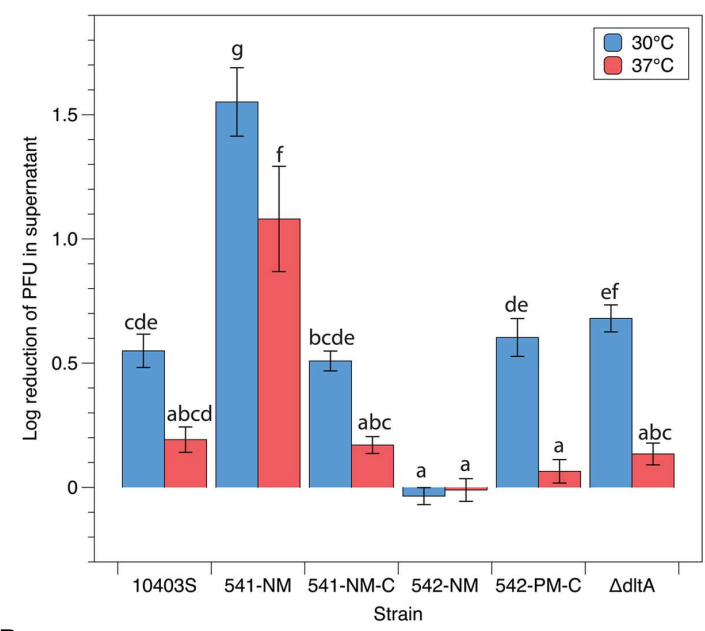

B

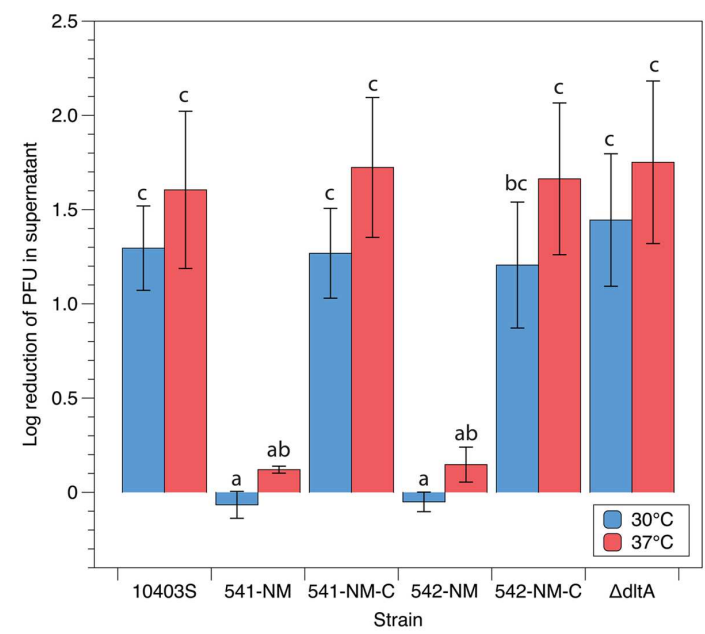

FIGURE 5 | Adsorption efficiencies of P100-like phages (A) LP-048 and (B) LP-125 on teichoic acid component mutants. Values shown are the $\log _{10}$ reduction of free phage in the supernatant of each co-incubation between phage and host. The $\log _{10}$ reduction is calculated by subtracting the log-transformed number of phage left in in the supernatant from the log-transformed number of phage in the negative control (BHI). We also tested the $\Delta$ dlttA strain complemented with the WT allele of dltA; however, no significant differences from either the $\Delta$ dltA strain or WT $10403 S$ were observed. Lower values represent less efficient adsorption. Bars that do not share any letters represent values that are significantly different; for example, a bar marked with $\mathrm{c}, \mathrm{d}$, and $\mathrm{e}$ is significantly different from a bar marked with $\mathrm{f}$. Three biological replicates were conducted. Error bars represent the standard error.

under standard laboratory growth temperatures for Listeria (e.g., 30 and $37^{\circ} \mathrm{C}$ ).

\section{Temperature Affects the Efficiency of Plaquing of P100-like Phages and P70-like Phages}

Here, we showed that the efficiencies of plaquing (EOP) of phages infecting L. monocytogenes are affected by temperature; these results are consistent with those found in other phagehost systems. For example, Sanders and Klaenhammer (1984) found that the EOP of the Streptococcus phage $\Phi 18$ was significantly affected by temperature, and specifically, showed that the growth temperature of the bacteria prior to infection had more of an effect on EOP than the incubation temperature post-infection. In another study, McConnell and Wright (1979) showed that Salmonella phage Felix-01 showed a higher EOP on a non-sensitive strains at incubation temperatures of 23 and $30^{\circ} \mathrm{C}$ than at 34,38 , and $42^{\circ} \mathrm{C}$. However, Ellis and Delbrück (1939) did not find any significant differenced in the EOP of an Escherichia phage under different growth temperatures $\left(37,24\right.$, and $\left.10^{\circ} \mathrm{C}\right)$; although, unlike in the experiments here and by Sanders and Klaenhammer (1984), Ellis and Delbrück (1939) did not acclimate their host bacteria to the experimental temperature prior to exposure to the phage.

Previously, Kim and Kathariou (2009) showed that L. monocytogenes Epidemic Clone II strains were resistant to phage infection at temperatures below $37^{\circ} \mathrm{C}$ and that phages A511 and 20422-1 were active on other L. monocytogenes strains (including serotype $1 / 2 \mathrm{a}, 1 / 2 \mathrm{~b}, 1 / 2 \mathrm{c}$, and $4 \mathrm{~b}$ strains) at $37^{\circ} \mathrm{C}$. In contrast, A511 did not form visible plaques at $37^{\circ} \mathrm{C}$ under the specific conditions used in our study (Figure 1). In a follow-up study, Kim et al. (2012) found that a restriction modification system was expressed at temperatures below $37^{\circ} \mathrm{C}$, and that deleting this restriction modification system restored phage susceptibility at $25^{\circ} \mathrm{C}$. In this study, we showed a very different pattern of temperature affecting the plaquing efficiency of $L$. monocytogenes. None of the phages tested in this study formed visible plaques at $37^{\circ} \mathrm{C}$, and all of the phages showed the highest EOP at temperatures under $21^{\circ} \mathrm{C}$. These results were consistent with much earlier observations that $37^{\circ} \mathrm{C}$ incubation temperature had a deactivating effect on temperate Listeria phages (Audurier et al., 1977). One possible explanation for the differences observed between our study and the study by Kim et al. (2012) could be that the phages we used were all isolated from silage in New York State (USA), whereas A511 and 20422-1 were isolated from sewage and a turkey processing plant, respectively. Therefore, the phages from silage may have adapted to cooler conditions than phages A511 and 20422-1 (silage is typically stored outdoors, and New York is a temperate climate); however, as we observed here that A511 showed the same plaquing phenotype as LP-125 against Mack and F2365 at 30 and $37^{\circ} \mathrm{C}$ (i.e., no plaquing was observed at $37^{\circ} \mathrm{C}$ ), future studies will be needed to test this.

\section{Adsorption Inhibition May Prevent Plaquing of LP-048, But Not of LP-125, at $37^{\circ} \mathrm{C}$}

Previously, both LP-048 and LP-125 were shown to have severe adsorption defects to L. monocytogenes strains with deleterious mutations in genes that are essential for rhamnosylation of WTA, which suggested that both phages use rhamnose as a phage receptor. However, LP-125 was shown to also 
require terminal $\mathrm{N}$-acetylglucosamine (GlcNAc) in the WTA for successful host adsorption (Denes et al., 2015). Thus, GlcNAc and rhamnose are likely the phage receptors for LP125 on serotype $1 / 2$ a strains, which is consistent with phages A511 (Habann et al., 2014) and P35 (Bielmann et al., 2015), both of which bind to GlcNAc and rhamnose. However, it should be noted that conclusions from Habann et al. (2014) differ from those from a prior study that identified peptidoglycan as A511's receptor (Wendlinger et al., 1996); this inconsistency is presumably due to limitations in the methodology used by Wendlinger et al. (1996) such as relying on cell fractions, that might have different structures exposed than those found on the surface of intact cells, to assess phage adsorption. Our observation that LP-048 has a severely reduced adsorption efficiency to L. monocytogenes at $37^{\circ} \mathrm{C}$ suggests that rhamnose expression in the WTA is down regulated at $37^{\circ} \mathrm{C}$. However, as LP-125 is still able to bind to L. monocytogenes at $37^{\circ} \mathrm{C}$ with similar efficiency as at $30^{\circ} \mathrm{C}$, there is likely still sufficient rhamnose present at $37^{\circ} \mathrm{C}$ to facilitate LP-125 binding to GlcNAc residues. Furthermore, our observation that LP-125 does still adsorb at $37^{\circ} \mathrm{C}$ suggests that there is an adsorption-independent mechanism of phage resistance that is preventing plaque formation at $37^{\circ} \mathrm{C}$. Whereas, we found no homologous genes to the previously described temperature dependent restrictionmodification system LmoH7 (Kim et al., 2012), it is possible that a restriction-modification system not homologous to $\mathrm{LmoH7}$ is present in the strains tested in this study. Alternatively, there could be an unrelated temperature dependent phagedefense system such as an abortive-infection system, CRISPRCas system, or super-infection exclusion system (Labrie et al., 2010).

We also showed that LP-125 could adsorb to the serotype $4 \mathrm{~b}$ strain $\mathrm{F} 2365$ at $30^{\circ} \mathrm{C}$, although significantly less efficiently than to serotype $1 / 2$ strains. This is likely because serotype $4 \mathrm{~b}$ strains do not possess rhamnose or terminal GlcNAc in their WTA. Instead, the WTA of serotype $4 \mathrm{~b}$ strains are decorated with terminal glucose and galactose (Fiedler et al., 1984; Fiedler, 1988), whereas the WTA of serotype 4 a strains are only decorated with terminal galactose (Fujii et al., 1985). As LP-125 binds to serotype $4 \mathrm{~b}$ strain F2365 but not to the serotype $4 \mathrm{a}$ strain J1-208, we hypothesize that glucose could be the phage receptor for LP-125 on serotype $4 \mathrm{~b}$ strains. Interestingly, we also showed that LP-125 has a significantly reduced adsorption efficiency on $\mathrm{F} 2365$ at $37^{\circ} \mathrm{C}$, as compared to $30^{\circ} \mathrm{C}$. This suggests that the surface receptor used by LP-125 on the serotype $4 \mathrm{~b}$ strain F2365 is either down regulated or less accessible at $37^{\circ} \mathrm{C}$.

\section{There Is Likely Competition between Rhamnose and GIcNAc Glycosylation of the WTA at Both 30 and $37^{\circ} \mathrm{C}$}

Our observation that the mutant strain lacking GlcNAc in its WTA (541-NM) allowed for significantly greater adsorption of LP-048 at both 30 and $37^{\circ} \mathrm{C}$ suggests that there is competition between the glycosylation of the WTA with rhamnose and
GlcNAc. Both glycosyl groups are linked to the WTA from the same hydroxyl sites on the polyol phosphate backbone (Fiedler, 1988). The mutant strain lacking GlcNAc WTA decorations would have more hydroxyl groups in the WTA available for rhamnosylation, which would explain the increased adsorption of LP-048 against this mutant strain. Given that we still see a significant difference in adsorption of LP048 against $541-\mathrm{NM}$ between 30 and $37^{\circ} \mathrm{C}$, we can rule out that the effect of temperature on LP-048 adsorption is solely due to differential regulation of GlcNAc glycosylation of the WTA; down-regulation of GlcNAc at $37^{\circ} \mathrm{C}$ is not increasing rhamnosylation of WTA at $37^{\circ} \mathrm{C}$. It is much more likely that rhamnosylation of the WTA is directly regulated in response to temperature. Although outside of the scope of this study, further research will be needed to elucidate the mechanisms putatively regulating the glycosyl decorations of Listeria's WTA.

\section{L. monocytogenes Can Gain Physiological Refuge from Phage Infection}

Physiological refuge can be defined as the ability of a phagesusceptible bacterium to gain transient resistance to phageinfection. The concept was postulated by Lenski (1988) to account for the stability of virulent phage and host populations. Previous studies have provided evidence that bacterial cells can take physiological refuge from phage-infection. For example, a number of studies have shown that adsorption rates of E. coli phages are affected by the physiological state of the host (Delbrück, 1940; Hadas et al., 1997; Chapman-McQuiston and Wu, 2008; Høyland-Kroghsbo et al., 2013). The susceptibility of the Firmicutes Lactococcus (Mullermerbach et al., 2007) and Lactobacillus (Marcó et al., 2015) to phage infection has also been shown to be affected by the physiological state of the host. A specific temperature dependent restriction-modification system has been shown to be present in a clonal group of L. monocytogenes strains, which demonstrated a specific case of L. monocytogenes taking physiological refuge (Kim et al., 2012). However, we have now shown that temperature generally affects L. monocytogenes' susceptibility to phage infection through both mechanisms of adsorption inhibition as well as unidentified internal (i.e., post-adsorption) mechanisms of phage resistance. The data presented here clearly shows that L. monocytogenes can gain physiological refuge from phage infection, which should be carefully considered for both the design and implementation of phage-based control and detection applications. These data also offer insight on the evolutionary dynamics of $L$. monocytogenes and its phages, which can help in our understanding of the diversity and transmission of this important foodborne pathogen.

\section{AUTHOR CONTRIBUTIONS}

TD and JT designed the experiments. MW provided reagents. JT performed the experiments. DK and TD performed the 
data analyses. TD prepared the figures. TD, JT, DK, and MW interpreted the data. TD and JT wrote the manuscript with valuable feedback from MW and DK.

\section{FUNDING}

This material is partially based upon work that is supported by the National Institute of Food and Agriculture, U.S. Department of Agriculture Hatch funds (NYC-143445) as well as a NIFA/USDA-AFRI Food safety Education Grant (NIFA 2010-04502), both to MW. Any opinions, findings, conclusions, or recommendations expressed in this publication are those of the authors and do not necessarily

\section{REFERENCES}

Altschul, S. F., Gish, W., Miller, W., Myers, E. W., and Lipman, D. J. (1990). Basic local alignment search tool. J. Mol. Biol. 215, 403-410. doi: 10.1016/S00222836(05) 80360-2

Audurier, A., Rocourt, J., and Courtieu, A. L. (1977). Isolement et caractérisation de bactériophages de Listeria monocytogenes. Ann. Microbiol. (Inst. Pasteur.) 128, 185-198.

Batz, M. B., Hoffmann, S., and Morris, J. G. (2012). Ranking the disease burden of 14 pathogens in food sources in the United States using attribution data from outbreak investigations and expert elicitation. J. Food Prot. 75, 1278-1291. doi: 10.4315/0362-028X.JFP-11-418

Bergholz, T. M., Bakker den, H. C., Fortes, E. D., Boor, K. J., and Wiedmann, M. (2010). Salt stress phenotypes in Listeria monocytogenes vary by genetic lineage and temperature. Foodborne Pathog. Dis. 7, 1537-1549. doi: $10.1089 /$ fpd.2010.0624

Bielmann, R., Habann, M., Eugster, M. R., Lurz, R., Calendar, R., Klumpp, J., et al. (2015). Receptor binding proteins of Listeria monocytogenes bacteriophages A118 and P35 recognize serovar-specific teichoic acids. Virology 477, 110-118. doi: 10.1016/j.virol.2014.12.035

Bishop, D. K., and Hinrichs, D. J. (1987). Adoptive transfer of immunity to Listeria monocytogenes. The influence of in vitro stimulation on lymphocyte subset requirements. J. Immunol. 139, 2005-2009.

Cartwright, E. J., Jackson, K. A., Johnson, S. D., Graves, L. M., Silk, B. J., and Mahon, B. E. (2013). Listeriosis outbreaks and associated food vehicles, United States, 1998-2008. Emerg. Infect. Dis. 19, 1-9. doi: 10.3201/eid1901.120393

Carvalho, F., Atilano, M. L., Pombinho, R., Covas, G., Gallo, R. L., Filipe, S. R., et al. (2015). L-Rhamnosylation of Listeria monocytogenes wall teichoic acids promotes resistance to antimicrobial peptides by delaying interaction with the membrane. PLoS Pathog. 11:e1004919. doi: 10.1371/journal.ppat.1004919

Chapman-McQuiston, E., and Wu, X. L. (2008). Stochastic receptor expression allows sensitive bacteria to evade phage attack. Part I: experiments. Biophys. J. 94, 4525-4536. doi: 10.1529/biophysj.107.120212

de Noordhout, C. M., Devleesschauwer, B., Angulo, F. J., Verbeke, G., Haagsma, J., Kirk, M., et al. (2014). The global burden of listeriosis: a systematic review and meta-analysis. Lancet Infect. Dis. 14, 1073-1082. doi: 10.1016/S14733099(14)70870-9

Delbrück, M. (1940). Adsorption of bacteriophage under various physiological conditions of the host. J. Gen. Physiol. 23, 631-642. doi: 10.1085/jgp.23.5.631

den Bakker, H. C., Bowen, B. M., Rodriguez-Rivera, L. D., and Wiedmann, M. (2012). FSL J1-208, a virulent uncommon phylogenetic lineage IV Listeria monocytogenes strain with a small chromosome size and a putative virulence plasmid carrying internalin-like genes. Appl. Environ. Microbiol. 78, 1876-1889. doi: 10.1128/AEM.06969-11

Denes, T., den Bakker, H. C., Tokman, J. I., Guldimann, C., and Wiedmann, M. (2015). Selection and characterization of phage-resistant mutant strains of Listeria monocytogenes reveal host genes linked to phage adsorption. Appl. Environ. Microbiol. 81, 4295-4305. doi: 10.1128/AEM.00087-15

Denes, T., Vongkamjan, K., Ackermann, H.-W., Moreno Switt, A. I., Wiedmann, M., and den Bakker, H. C. (2014). Comparative genomic and reflect the view of the U.S. Department of Agriculture References.

\section{ACKNOWLEDGMENT}

We thank Hélène Marquis for kindly gifting us the $\Delta$ dltA mutant.

\section{SUPPLEMENTARY MATERIAL}

The Supplementary Material for this article can be found online at: http://journal.frontiersin.org/article/10.3389/fmicb. 2016.00631

morphological analyses of Listeria phages isolated from farm environments. Appl. Environ. Microbiol. 80, 4616-4625. doi: 10.1128/AEM.00720-14

Denes, T., and Wiedmann, M. (2014). Environmental responses and phage susceptibility in foodborne pathogens: implications for improving applications in food safety. Curr. Opin. Biotechnol. 26, 45-49. doi: 10.1016/j.copbio.2013.09.001

Ellis, E. L., and Delbrück, M. (1939). The growth of bacteriophage. J. Gen. Physiol. 22, 365-384. doi: 10.1085/jgp.22.3.365

Fiedler, F. (1988). Biochemistry of the cell surface of Listeria strains: a locating general view. Infection 16(Suppl. 2), S92-S97. doi: 10.1007/BF01639729

Fiedler, F., Seger, J., Schrettenbrunner, A., and Seeliger, H. P. R. (1984). The biochemistry of murein and cell wall teichoic acids in the genus Listeria. Syst. Appl. Microbiol. 5, 360-376. doi: 10.1016/S0723-2020(84)80038-7

Fujii, H., Kamisango, K., Nagaoka, M., Uchikawa, K., Sekikawa, I., Yamamoto, K., et al. (1985). Structural study on teichoic acids of Listeria monocytogenes types 4a and 4d. J. Biochem. 97, 883-891.

Habann, M., Leiman, P. G., Vandersteegen, K., Van den Bossche, A., Lavigne, R., Shneider, M. M., et al. (2014). Listeria phage A511, a model for the contractile tail machineries of SPO1-related bacteriophages. Mol. Microbiol. 92, 84-99. doi: 10.1111/mmi.12539

Hadas, H., Einav, M., Fishov, I., and Zaritsky, A. (1997). Bacteriophage T4 development depends on the physiology of its host Escherichia coli. Microbiology 143, 179-185. doi: 10.1099/00221287-143-1-179

Hodgson, D. A. (2000). Generalized transduction of serotype 1/2 and serotype $4 \mathrm{~b}$ strains of Listeria monocytogenes. Mol. Microbiol. 35, 312-323. doi: 10.1046/j.1365-2958.2000.01643.x

Høyland-Kroghsbo, N. M., Mærkedahl, R. B., and Svenningsen, S. L. (2013). A quorum-sensing-induced bacteriophage defense mechanism. MBio 4, e00362-12. doi: 10.1128/mBio.00362-12

Kang, J., Stasiewicz, M. J., Murray, D., Boor, K. J., Wiedmann, M., and Bergholz, T. M. (2014). Optimization of combinations of bactericidal and bacteriostatic treatments to control Listeria monocytogenes on cold-smoked salmon. Int. J. Food Microbiol. 179, 1-9. doi: 10.1016/j.ijfoodmicro.2014.03.017

Kim, J.-W., Dutta, V., Elhanafi, D., Lee, S., Osborne, J. A., and Kathariou, S. (2012). A novel restriction-modification system is responsible for temperaturedependent phage resistance in Listeria monocytogenes ECII. Appl. Environ. Microbiol. 78, 1995-2004. doi: 10.1128/AEM.07086-11

Kim, J.-W., and Kathariou, S. (2009). Temperature-dependent phage resistance of Listeria monocytogenes epidemic clone II. Appl. Environ. Microbiol. 75, 2433-2438. doi: 10.1128/AEM.02480-08

Kropinski, A. M., Mazzocco, A., Waddell, T. E., Lingohr, E., and Johnson, R. P. (2009). Enumeration of bacteriophages by double agar overlay plaque assay. Methods Mol. Biol. 501, 69-76. doi: 10.1007/978-1-60327-164-6_7

Labrie, S. J., Samson, J. E., and Moineau, S. (2010). Bacteriophage resistance mechanisms. Nat. Rev. Microbiol. 8, 317-327. doi: 10.1038/nrmicro2315

Lenski, R. E. (1988). "Dynamics of interactions between bacteria and virulent bacteriophage," in Advances in Microbial Ecology, ed. K.C. Marshall (New York, NY: Springer US), 1-39. doi: 10.1007/978-1-4684-5409-3_1

Lenth, R. V., and Hervé, M. (2014). lsmeans: Least-Squares Means. R Package (Version 2.18). Available at: http://CRAN.R-project.org/package=lsmeans 
Linnan, M. J., Mascola, L., Lou, X. D., Goulet, V., May, S., Salminen, C., et al. (1988). Epidemic listeriosis associated with Mexican-style cheese. N. Engl. J. Med. 319, 823-828. doi: 10.1056/NEJM198809293191303

Loessner, M. J., and Busse, M. (1990). Bacteriophage typing of Listeria species. Appl. Environ. Microbiol. 56, 1912-1918.

Marcó, M. B., Reinheimer, J., and Quiberoni, A. (2015). Phage adsorption and lytic propagation in Lactobacillus plantarum: could host cell starvation affect them? BMC Microbiol. 15:273. doi: 10.1186/s12866-0150607-1

McConnell, M., and Wright, A. (1979). Variation in the structure and bacteriophage-inactivating capacity of Salmonella anatum lipopolysaccharide as a function of growth temperature. J. Bacteriol. 137, $746-751$.

Mullermerbach, M., Kohler, K., and Hinrichs, J. (2007). Environmental factors for phage-induced fermentation problems: replication and adsorption of the Lactococcus lactis phage P008 as influenced by temperature and pH. Food Microbiol. 24, 695-702. doi: 10.1016/j.fm.2007.04.003

R Core Team (2009). R: A Language, and Environment for Statistical Computing. Vienna: R Foundation for Statistical Computing.

Sanders, M. E., and Klaenhammer, T. R. (1984). Phage resistance in a phage-insensitive strain of Streptococcus lactis: temperature-dependent phage development and host-controlled phage replication. Appl. Environ. Microbiol. 47, 979-985.

Scallan, E., Hoekstra, R. M., Angulo, F. J., Tauxe, R. V., Widdowson, M.A., Roy, S. L., et al. (2011). Foodborne illness acquired in the United States-major pathogens. Emerg. Infect. Dis. 17, 7-15. doi: 10.3201/eid1701. P11101

Stasiewicz, M. J., Wiedmann, M., and Bergholz, T. M. (2010). The combination of lactate and diacetate synergistically reduces cold growth in brain heart infusion broth across Listeria monocytogenes lineages. J. Food Prot. 73, $631-640$.
Strydom, A., and Witthuhn, C. R. (2015). Listeria monocytogenes: a target for bacteriophage biocontrol. Compr. Rev. Food Sci. Food Saf. 14, 694-704. doi: 10.1111/1541-4337.12153

Vazquez-Boland, J. A., Kuhn, M., Berche, P., Chakraborty, T., DominguezBernal, G., Goebel, W., et al. (2001). Listeria pathogenesis and molecular virulence determinants. Clin. Microbiol. Rev. 14, 584-640. doi: 10.1128/CMR.14.3.584-640.2001

Vongkamjan, K., Switt, A. M., Bakker den, H. C., Fortes, E. D., and Wiedmann, M. (2012). Silage collected from dairy farms harbors an abundance of listeriaphages with considerable host range and genome size diversity. Appl. Environ. Microbiol. 78, 8666-8675. doi: 10.1128/AEM.01859-12

Wendlinger, G., Loessner, M. J., and Scherer, S. (1996). Bacteriophage receptors on Listeria monocytogenes cells are the $\mathrm{N}$-acetylglucosamine and rhamnose substituents of teichoic acids or the peptidoglycan itself. Microbiology 142, 985-992. doi: 10.1099/00221287-142-4-985

Wesley, I. V., and Ashton, F. (1991). Restriction enzyme analysis of Listeria monocytogenes strains associated with food-borne epidemics. Appl. Environ. Microbiol. 57, 969-975.

Wickham, H. (2007). Reshaping data with the reshape package. J. Stat. Softw. 21, 1-20. doi: 10.3978/j.issn.2305-5839.2016.01.33

Conflict of Interest Statement: MW serves as a scientific advisor for and has a financial interest in Sample6, a company that is producing phage-based diagnostics for foodborne pathogens.

Copyright (c) 2016 Tokman, Kent, Wiedmann and Denes. This is an open-access article distributed under the terms of the Creative Commons Attribution License (CC BY). The use, distribution or reproduction in other forums is permitted, provided the original author(s) or licensor are credited and that the original publication in this journal is cited, in accordance with accepted academic practice. No use, distribution or reproduction is permitted which does not comply with these terms. 